\title{
Late Blockade of T Cell Costimulation Interrupts Progression of Experimental Chronic Allograft Rejection
}

\author{
Anil Chandraker, ${ }^{\star}$ Haruhito Azuma, ${ }^{\ddagger}$ Kari Nadeau, ${ }^{\ddagger}$ Charles B. Carpenter, ${ }^{\star}$ Nicholas L. Tilney, ${ }^{\ddagger}$ Wayne W. Hancock, ${ }^{\S}$ \\ and Mohamed H. Sayegh* \\ $*$ Laboratory of Immunogenetics and Transplantation, and ${ }^{\ddagger}$ Department of Surgery, Brigham and Women’s Hospital; and ${ }^{\S}$ Department of \\ Pathology, Beth Israel Deaconess Medical Center, Harvard Medical School, Boston, Massachusetts 02115
}

\begin{abstract}
Early blockade of $\mathrm{T}$ cell-costimulatory activation pathways prevents development of experimental chronic allograft rejection. Ongoing $T$ cell recognition of alloantigen and activation may also play an important role in progression of chronic rejection, but definitive evidence is lacking. We used the fusion protein CTLA4Ig to block CD28-B7 T cell costimulation late after the onset of initial graft injury. Using the F334 into LEW rat model of chronic renal allograft rejection, transplant recipients were treated with a $10-d$ course of cyclosporine, and a subgroup received a single injection of CTLA4Ig at $8 \mathrm{wk}$ after transplant.

Functionally, CTLA4Ig administration prevented development of progressive proteinuria $(14.3 \pm 4.1 \mathrm{mg} / 24 \mathrm{~h}$ versus $41.0 \pm 12.0 \mathrm{mg} / 24 \mathrm{~h}$ at $24 \mathrm{wk}$ after transplant, $P<0.05)$. Histologically, graft mononuclear cell infiltration, glomerular hypertrophy, focal and segmental glomerulosclerosis, and intimal vascular hyperplasia were all attenuated in CTLA4Igtreated animals. Lastly, reverse transcriptase-PCR and immunohistologic studies showed a significant reduction in the intragraft expression of key products of $\mathrm{T}$ cell and macrophage activation, and upregulation of what have recently been termed as "protective" genes, including the bcl family members, Bcl-2 and Bcl-xL, and hemoxygenase.

Our data are the first to demonstrate that blocking $T$ cell-costimulatory activation late after transplantation, after initial graft injury, prevents progression of chronic allograft rejection supporting the hypothesis that ongoing $T$ cell recognition of alloantigen and activation are key mediators of ongoing chronic allograft rejection. (J. Clin. Invest. 1998. 101:2309-2318.) Key words: CD28 • B7 • CTLA4Ig • rat $\cdot$ transplantation
\end{abstract}

\section{Introduction}

The pathogenesis of chronic rejection and the mechanisms responsible for its progression remain obscure, despite it being

Mohamed H. Sayegh and Wayne W. Hancock are co-senior authors. Address correspondence to Mohamed H. Sayegh, Laboratory of Immunogenetics and Transplantation, Brigham and Women's Hospital, 75 Francis Street, Boston, MA 02115. Phone: 617-732-5259; FAX: 617-732-5254; E-mail: sayegh@bustoff.bwh.harvard.edu

Received for publication 19 November 1997 and accepted in revised form 20 March 1998.

J. Clin. Invest.

(C) The American Society for Clinical Investigation, Inc. 0021-9738/98/06/2309/10 \$2.00

Volume 101, Number 11, June 1998, 2309-2318

http://www.jci.org the most common cause of long-term allograft loss (1-3). It has been suggested that both alloantigen-dependent and alloantigen-independent factors play an important role in the pathogenesis of chronic rejection (4). The clinical observations that acute allograft rejection and delayed graft function are major risk factors for the development of chronic rejection led to the concept that initial graft damage sets into motion a series of potentially irreversible events, resulting ultimately in allograft failure. Experimental evidence to support this comes from reports which indicate that prevention of acute rejection and induction of tolerance may prevent development of chronic rejection (5-8). In particular, blocking $\mathrm{T}$ cell-costimulatory activation early after transplantation prevents development of experimental chronic cardiac (9-11), as well as renal (12), allograft rejection. It has been suggested, however, that continuous $T$ cell recognition of alloantigen, presumably via the indirect pathway (i.e., $\mathrm{T}$ cells that recognize donor-derived peptides presented by self antigen-presenting cells [APCs] $]^{1}$ ), and activation may play an important role in progression of chronic rejection (13), although definitive studies are lacking. Understanding these mechanisms is essential for development of clinical strategies to prevent and interrupt progression of chronic allograft rejection.

The F344 to LEW rat renal allograft model is a well established model for the study of experimental chronic allograft rejection. In this model, kidneys are orthotopically transplanted and the animals are treated with a short course of low dose cyclosporine (CsA) to reverse acute rejection. If left without further intervention, these animals develop classical features of chronic rejection: namely, progressive proteinuria, graft interstitial mononuclear cell infiltration, arteriosclerosis, and glomerulosclerosis $(12,14)$. These functional and pathologic features are associated with intragraft expression of various activation and inflammatory cytokines and growth factors that have been associated with development of the chronic rejection process (14-16).

The purpose of this study was to investigate the role of the $\mathrm{T}$ cell activation in the progression of chronic rejection after initial graft injury had occurred. It is currently accepted that, in order for T cells to be fully activated, they require two signals: signal 1 is delivered after $\mathrm{T}$ cells recognize antigen through $\mathrm{T}$ cell receptor (TCR) interaction with an MHC-peptide complex on APCs. Signal 2, a costimulatory signal, is delivered by interaction of $\mathrm{T}$ cell receptors with their specific ligands on APCs. Perhaps the best characterized T cell-costimulatory signal is that delivered by CD28 binding to B7 $(13,17-20)$; blockade of this pathway prevents acute graft rejection and induces

1. Abbreviations used in this paper: APC, antigen-presenting cell; CSA, cyclosporine; HO-1, hemoxygenase-1; PAP, peroxidase-antiperoxidase complex; PAS, periodic acid-Schiff; RT, reverse transcriptase; TCR, T cell receptor. 
tolerance in some transplant models $(13,21-27)$. More recently, we have shown that early blockade of CD28-B7 by CTLA4Ig prevents development of chronic rejection $(9,12)$. In the present study, we used the fusion protein CTLA4Ig to block CD28-B7 T cell costimulation several weeks after transplantation, after the initial injury occurred. The results support the hypothesis that ongoing $\mathrm{T}$ cell recruitment and activation play a key role in the progression of chronic rejection. They also provide the rationale to develop novel strategies to treat chronic rejection, a clinical problem that at the current time has no specific therapy.

\section{Methods}

Animals. Inbred 200-250 g male LEW rats (LEW, RT1 ${ }^{1}$ ) were transplanted with kidneys from Fischer 344 (F344, RT1 $1^{\text {lv1 }}$ ) rats (allografts) or LEW rats (isografts); animals were purchased from Harlan SpragueDawley (Indianapolis, IN).

Kidney transplantation. Kidneys were transplanted orthotopically to the left renal vessels and the left ureter of the host by end-toend anastomosis using $10-0$ prolene sutures. The right kidney was removed $10 \mathrm{~d}$ later, as described (12).

Reagents. The human fusion protein CTLA4Ig was obtained from Bristol-Myers-Squibb (Seattle, WA). CsA was obtained from Sandoz Research Institute (East Hanover, NJ).

Experimental groups. The control group $(n=13)$ of LEW rats received F344 kidneys and a short course of CsA $(5 \mathrm{mg} / \mathrm{kg} / \mathrm{d} \times 10 \mathrm{~d}$, subcutaneously) and no further treatment. The treated group $(n=$ 12) received the same CsA protocol, as well as a single injection of CTLA4Ig ( $0.5 \mathrm{mg}$, intraperitoneally) at 8 wk postoperatively. Another group of animals treated with a single intraperitoneal injection of CTLA4Ig at 16 wk was also set up $(n=4)$. Grafts $(n=3-4)$ were harvested from randomly selected animals of the different groups at 16 or 24 wk after transplant, for morphologic, immunohistologic, and molecular analyses.

Function. Urine $(24 \mathrm{~h})$ was collected every $2-4 \mathrm{wk}$ from the time of transplantation. Protein excretion was determined by measuring precipitation after interaction with $3 \%$ sulfosalicylic acid. Turbidity was assessed by absorbance at a wavelength of $595 \mathrm{~nm}$ using a Coleman Junior II spectrophotometer (12).

Morphology. Kidney grafts from each recipient group $(n=3-4 /$ group/time point) were fixed in $10 \%$ buffered formalin, embedded in paraffin, sectioned and stained with hematoxylin and eosin for evaluation of cellular infiltrates by light microscopy, or stained by periodic acid-Schiff (PAS) for evaluation of glomerulosclerosis and vascular intimal hyperplasia (14).

Immunopathology. mAbs, obtained from Serotec (Harlan Bioproducts for Science, Indianapolis, IN), except where noted, were directed against all rat leukocytes (CD45); T cells (TCR- $\alpha / \beta, \mathrm{R} 73)$; and mononuclear phagocytes (CD68, ED-1). Cellular activation was assessed using mAbs to rat IL-2R, and the cytokines IL-2, IL-4, IL-10, IFN- $\gamma$, RANTES, and MCP-1 (Peprotech, Wallingford, CT), plus monospecific polyclonal antibodies to IL-13, TGF- $\beta$, PDGF, and TNF- $\alpha$ (R\&D Systems, Minneapolis, MN). Humoral responses were analyzed using rabbit anti-rat IgM, IgG, C3, and fibrin (ICN, Irvine, CA). Expression of pro-apoptotic genes (including CPP-32, also known as YAMA or capase-3, Bad, and ICH-1L) and antiapoptotic genes (Bcl-2, Bcl-xL) was determined using mAbs obtained from Transduction Laboratories (Lexington, KY); rabbit anti-rat hemoxygenase-1 (HO-1) was purchased from StressGen (Victoria, British Columbia, Canada). mAb binding was detected using rat Ig-absorbed goat anti-mouse IgG (Sigma Chemical Co., St. Louis, MO), rabbit anti-goat IgG, and goat peroxidase-antiperoxidase complexes (PAP). Binding of rabbit or goat Abs was detected using swine antirabbit IgG and rabbit PAP, or rabbit anti-goat IgG and goat PAP, respectively, purchased from DAKO (Carpinteria, CA).
Grafts were harvested, subdivided, and quick-frozen in isopentane and stored at $-80^{\circ} \mathrm{C}$ in preparation for immunohistologic studies. Cryostat sections were fixed in paraformaldehyde-lysine-periodate for demonstration of leukocytes and activation antigens, or fixed in acetone for localization of cytokines, and stained by a three-layer (for polyclonal $\mathrm{Ab}$ ) or four-layer (for $\mathrm{mAb}$ ) PAP method, as previously described $(9,14,27)$. Briefly, sections were incubated overnight with primary antibodies at $4^{\circ} \mathrm{C}$, followed by incubations at room temperature with bridging antibodies, PAP complexes, and diaminobenzidine. Isotype-matched $\mathrm{mAbs}$ or purified Ig, and a control for residual endogenous peroxidase activity, were included in each experiment. Where indicated, antibody absorption with a corresponding cytokine (e.g., TGF- $\beta$, from R\&D) was undertaken before immunohistologic labeling (14). Numbers of labeled leukocytes within 10 consecutive high power fields of renal cortex/cryostat section/rat ( $n=4 /$ group) were determined, and compared by the unpaired, two-tailed Student's $t$ test. Cytokine and endothelial labeling was judged semiquantitatively, due to the presence of extracellular (cytokines) or continuous (e.g., endothelial cell) labeling, as negative, $<5 \%, 5-20 \%, 20-50 \%$, $50-90 \%$, or $>90 \%$ of the cells indicated, as described $(14,27)$.

Reverse transcriptase ( $R T)$-PCR analysis. RNA was isolated from frozen kidneys using a guanidinium isothiocyanate/phenol-chloroform isolation method (ULTRA-spec., Biotecx, Houston, TX). Total RNA was used for first strand cDNA using oligo $\mathrm{dT}_{12-18}$ and RT according to supplier-recommended conditions (GIBCO-BRL, Gaithersburg, MD). Nonlooping, nonoverlapping, oligonucleotide primer pairs from separate exons were prepared for each gene studied (IL-2, IFN- $\gamma$, IL-6, IL-12, TNF- $\alpha$, RANTES, MCP-1, TGF- $\beta$ ) by Clontech (Palo Alto, CA) or by Genosys (The Woodlands, TX). Competitive PCR for quantification of mRNA was performed as described (16). Amplification was begun with incubation at $94^{\circ} \mathrm{C}$ for $2 \mathrm{~min}$, and was optimized for each cytokine. PCR products $(5 \mathrm{ml})$ were run on an ethidium bromide-stained $1.5 \%$ agarose gel and gene-specific bands were visualized with ultraviolet light. We performed control experiments for each primer pair by substituting water for cDNA and omitting RT during the cDNA synthesis. Quantities of MIMIC or competitor fragments (Clontech) and target cDNA were compared using a PC SCANJET with analysis by Adobe Photoshop software (Adobe Inc., San Jose, CA). Absolute amounts of cytokine PCR products as determined by competitive PCR were corrected by dividing by amounts of $\beta$ actin PCR product. Each sample was repeated twice and values are expressed as mean \pm SD $(12,16,28,29)$.

Statistical analysis. For urinary protein excretion, one way ANOVA was performed at each time point. The group effects were all significant at the 0.05 level for $P$ values. To examine individual differences further, comparison between every pair of groups was performed. The two sample $t$ tests for independent samples were used for data from the RT-PCR analysis.

\section{Results}

Graft function. 36 animals were transplanted, 6 of which died by day 16 before the animals were randomly assigned to receive no further treatment $(n=18)$ or CTLA4Ig at 8 wk after transplant $(n=12)$. One animal in the control group died at 20 wk and another developed severe hematuria and was excluded from the analysis. A total of $11 \mathrm{LEW}$ into LEW isografts was also set up. Proteinuria has been reported to be one of the best surrogates for chronic kidney dysfunction in the F344 into LEW model of chronic renal allograft rejection (15). As the interruption group did not receive CTLA4Ig until 8 wk after transplantation, the protein excretion for all animals was graphed together as treatment up to this time was identical (Fig. 1). After this time point, the proteinuria was plotted separately. Animals that received CTLA4Ig were prospectively assigned at the time of operation to avoid any bias in animal 


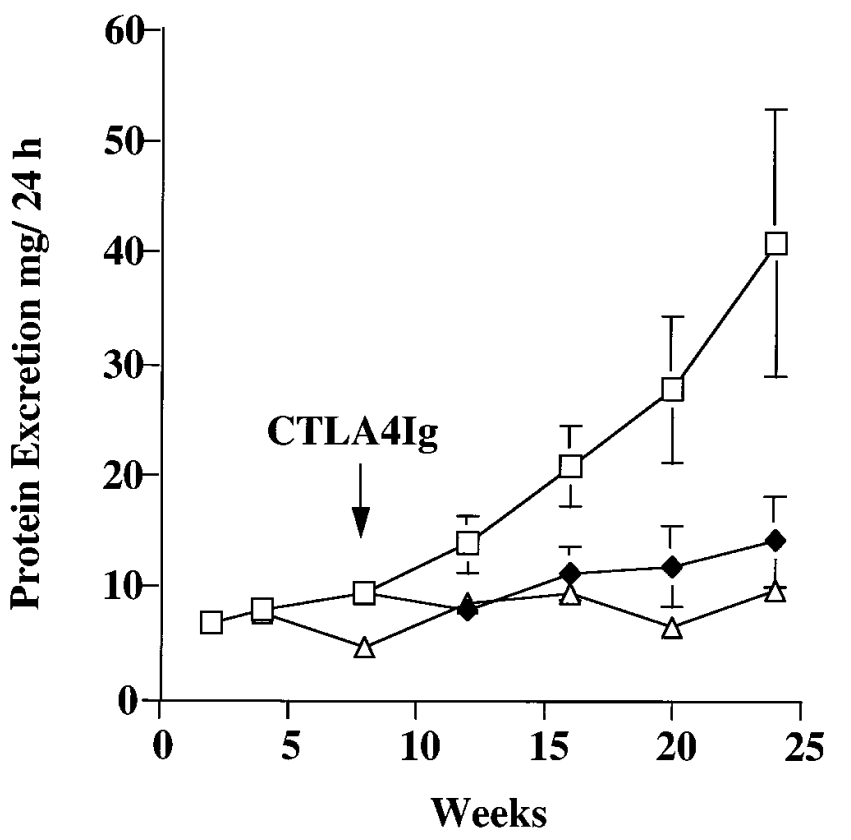

selection. In allograft recipients treated with CsA alone, animals usually start to show an increase in their 24-h protein excretion after 8-12 wk after transplantation $(12,14)$. In our study, animals treated with CsA plus CTLA4Ig at 8 wk after transplant did not develop progressive proteinuria throughout the follow-up period of $24 \mathrm{wk}(14.3 \pm 4.1 \mathrm{mg} / 24 \mathrm{~h}$ versus $41.0 \pm 12.0 \mathrm{mg} / 24 \mathrm{~h}$ at $24 \mathrm{wk}, P=0.043)$. Control isograft proteinuria was $9.88 \pm 0.68 \mathrm{mg} / 24 \mathrm{~h}$ at the same time point (Fig. 1). These data indicate that CD28-B7 blockade late after transplantation interrupts progressive deterioration in allograft function characteristic of chronic rejection in this model.

Graft morphology. At 8 wk after transplantation, the time at which CTLA4Ig was administered, allografts showed moderate to large perivascular and periglomerular aggregates of mononuclear cell infiltrates, but little morphologic evidence of renal injury, as previously noted (14). By $24 \mathrm{wk}$, the morphologic features of allografts in rats receiving CTLA4Ig were markedly different in comparison to rats given CsA alone (Fig. 2 ), based on analysis of paraffin sections $(4 \mu \mathrm{m})$ prepared from at least six different levels from the renal cortex of each of four grafts/group. Allografts in CsA-treated animals displayed the characteristic changes of chronic rejection: $79 \%$ of renal arteries $(n=126$ vessels studied) showed evidence of intimal proliferation with $20-50 \%$ luminal occlusion of individual vessels; $83 \%$ of glomeruli ( $n=213$ glomeruli studied) had focal and segmental proliferation, and dense interstitial infiltration by mononuclear cells, with large perivascular and periglomerular aggregates. By contrast, allografts within animals receiving additional therapy with CTLA4Ig at 8 wk showed only minor, nonspecific changes over those seen at $8 \mathrm{wk}$ : arteries $(n=136)$ showed evidence of ballooning of the endothelial cells and medial vacuolization, but only $3 \%$ of arteries showed any degree of intimal proliferation; glomeruli $(n=173)$ were normal apart from focal and mild to moderate hypercellularity of mesangial areas due to the glomerular accumulation of small numbers of mononuclear cells; and only minor focal interstitial infiltrates of mononuclear cells were noted. These findings clearly indi-

\section{$-\square-$ Control $\longrightarrow$ CTLA4Ig at $8 \mathrm{wk}$} Isograft

cate that CD28-B7 blockade late after transplantation interrupts progression of key morphological features of chronic rejection in this model.

Immunopathology. By $24 \mathrm{wk}$, allografts in CsA-treated recipients contained dense mononuclear cell infiltrates, consisting of macrophages and some T cells, and widespread evidence of cellular and humoral immune activation (Fig. 3; Table I). These grafts contained marked expression of the chemokines, RANTES and MCP-1, a largely Th1-predominant pattern of cytokine expression (IL-2, IFN- $\gamma$, and TNF- $\alpha$, but little or no IL-4, IL-10, or IL-13), and florid staining for the pro-fibrotic cytokines, TGF- $\beta$ and PDGF. Grafts also contained dense deposition of $\mathrm{IgG}, \mathrm{IgM}, \mathrm{C} 3$, and fibrin throughout the vasculature.

In contrast to the CsA group, allografts in animals receiving CsA plus CTLA4Ig showed a marked attenuation of both cellular and humoral responses (Table I). Addition of CTLA4Ig significantly reduced allograft infiltration by macrophages and T cells, as well as the extent of cellular IL-2R expression (Table I). CTLA4Ig largely reduced to background levels of intragraft staining for Th1 cytokines, chemokines, and pro-fibrotic cytokines, but was associated with an enhanced expression of all three Th2 cytokines examined (IL-4, IL-10, IL-13). CTLA4Ig also markedly diminished the extent of intragraft deposition of alloantibodies, and associated C3 and fibrin.

In recent studies of long-surviving grafts (30-32), the presence of "protective" genes that inhibit apoptosis, oxidative stress, and activation of NF-кB (33) was associated with infiltration of Th2 cytokine-producing cells and preservation of normal tissue architecture, whereas chronic rejection, including development of transplant arteriosclerosis, was linked with an absence of protective gene expression and upregulation of multiple pro-apoptotic genes, including CPP-32 and Bad. In the current study, negligible expression of any of these genes was apparent at $8 \mathrm{wk}$ after transplant (not shown), whereas marked differences in their expression was apparent at $24 \mathrm{wk}$ 


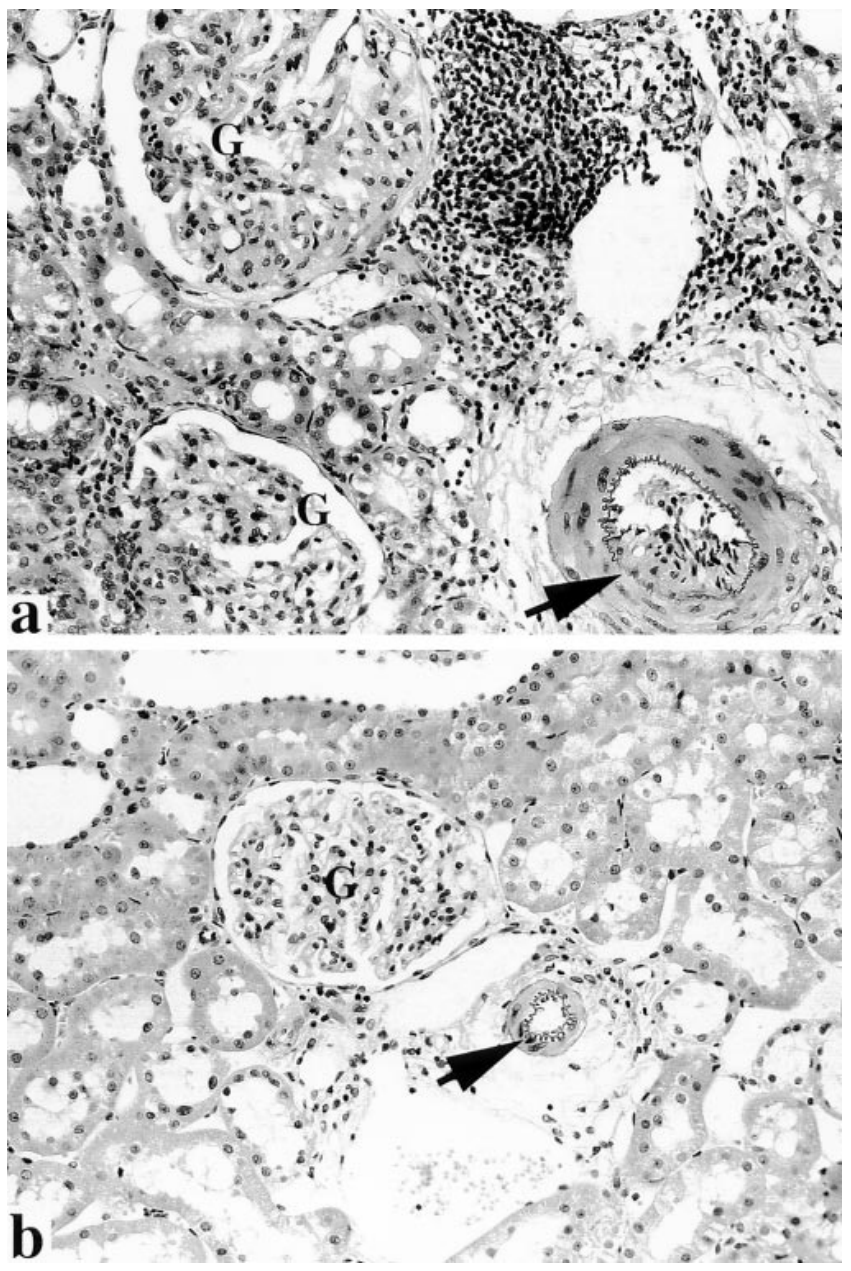

Figure 2. Graft histology at $24 \mathrm{wk}$. Representative histologic findings in paraffin sections of rat renal allografts harvested at $24 \mathrm{wk}$ after transplant from $(a)$ control animals and $(b)$ those which received CTLA4Ig at 8 wk. Glomeruli $(G)$ in control grafts show hypertrophy, focal and segmental proliferation, and focal and segmental sclerosis. Controls also show moderate to large periglomerular and perivascular mononuclear cell aggregates, tubular vacuolization and atrophy, and transplant arteriosclerosis; arrows indicate the internal elastic lamina, such that in $a$ there is $>50 \%$ occlusion of the lumen of an interlobular artery by the expanded intima. By contrast, CTLA4Ig administration resulted in preservation of normal glomerular architecture, tubular morphology and normal arteries, and was associated with only a low-level, diffuse mononuclear cell infiltrate. Representative of three to four animals/group. H\&E counterstain, $\times 75$.

Figure 3. Graft immunohistology at $24 \mathrm{wk}$. Representative immunohistologic findings ( $n=3 /$ group) in cryostat sections of rat renal allografts harvested at $24 \mathrm{wk}$ after transplant from control animals (left) and those which received CTLA4Ig at $8 \mathrm{wk}($ right); where possible, serial sections are shown containing glomeruli $(G)$, medium-sized arteries, and areas of intimal proliferation (arrows). In control grafts, sparse mononuclear cell labeling for IL-2 and moderate labeling for IFN- $\gamma$ are seen, whereas IL- 4 and IL-10 are essentially absent, and dense leukocyte and intimal labeling for TNF- $\alpha$, PDGF-A $\alpha$, and TGF- $\beta$ are seen. By contrast with the results in controls, CTLA4Ig therapy led to reduction in staining for IL-2, IFN- $\gamma$, TNF, PDGF-A $\alpha$, and TGF- $\beta$, whereas expression of IL- 4 and IL- 10 was increased. Hematoxylin counterstain, $\times 50$.

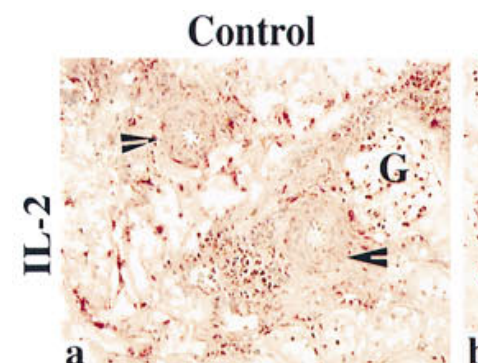

CTLA4Ig@ 8 wk



b
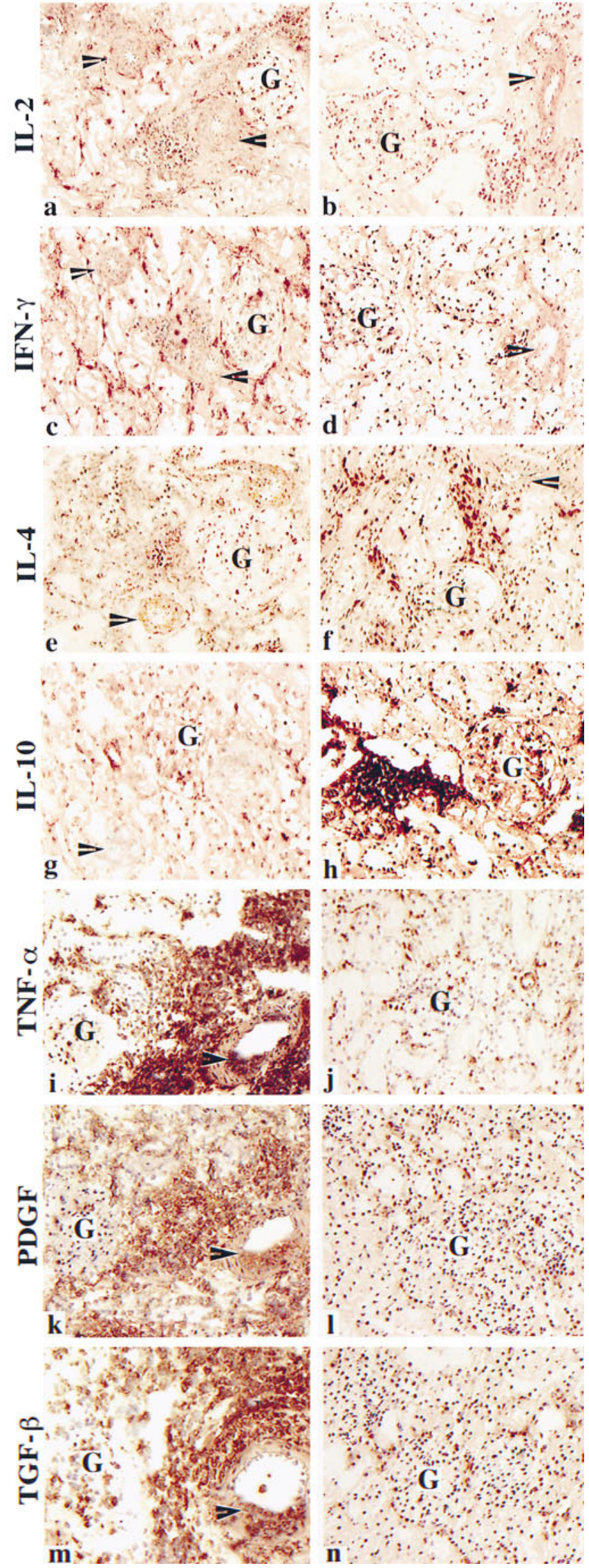
Table I. Delayed Administration of CTLA4Ig Attenuates Intragraft Leukocyte Infiltration, Expression of Pro-inflammatory Mediators, and Deposition of Humoral Reactants

\begin{tabular}{|c|c|c|}
\hline & CsA & CsA plus CTLA4Ig (at $8 \mathrm{wk}$ ) \\
\hline $\mathrm{CD} 45^{+}$leukocytes & $86 \pm 17$ & $37 \pm 11 *$ \\
\hline $\mathrm{TCR}^{+}$cells & $32 \pm 14$ & $14 \pm 9 *$ \\
\hline Macrophages & $51 \pm 23$ & $19 \pm 11 *$ \\
\hline IL- $2 \mathrm{R}^{+}$cells & $11 \pm 7$ & $3 \pm 2 *$ \\
\hline IL-2 & $5-20 \%$ leukocytes & Negative \\
\hline IFN- $\gamma$ & $5-20 \%$ leukocytes & Negative \\
\hline TNF- $\alpha$ & $>50 \%$ leukocytes, adjacent EC, SMC, and proximal tubules & $<5 \%$ leukocytes, focal EC \\
\hline TGF- $\beta$, PDGF & $20-50 \%$ leukocytes & $<5 \%$ leukocytes \\
\hline RANTES, MCP-1 & $5-20 \%$ leukocytes plus focal EC & Negative \\
\hline IL-4, IL-13 & $<5 \%$ leukocytes & $10-20 \%$ leukocytes, focal EC \\
\hline IL-10 & $<5 \%$ leukocytes & $20-50 \%$ leukocytes \\
\hline IgG, IgM, C3 & $3^{+}$all EC, all glomeruli & $1^{+}$focal EC, $<20 \%$ glomeruli \\
\hline Fibrin & $3^{+}$all EC and $>50 \%$ leukocytes & $1^{+}$focal EC, $<5 \%$ leukocytes \\
\hline Bcl-2, Bcl-xL, HO-1 & Rare interstitial leukocyte & $\begin{array}{l}\text { 10-20\% leukocytes plus vascular EC and SMC, } \\
\text { including glomerular cells }\end{array}$ \\
\hline CPP-32, Bad, ICH-1L & 20-50\% leukocytes, plus vascular EC and SMC & Focal vascular SMC and fibroblasts \\
\hline
\end{tabular}

Comparison of allografts at $24 \mathrm{wk}$ after transplant in animals $(n=4 /$ group $)$ given CsA alone versus CsA plus a single injection of CTLA4Ig at 8 wk; cell counts are expressed as mean \pm SD of positive cells/field of view (10 fields/section/kidney); $E C$, endothelial cells; $S M C$, smooth muscle cells. * $P<$ 0.0001 when compared with CsA (control) group using the Student's $t$ test.

(Table I; Fig. 4). The control group showed endothelial and smooth muscle expression of CPP-32 and Bad, and only minor expression of Bcl-2, Bcl-xL, or HO-1. By contrast, the use of CTLA4Ig at 8 wk led to expression of Bcl-2, Ho-1, and Bcl-xL (Fig. 4) at $24 \mathrm{wk}$, but only focal expression of the CPP-32 (Fig. 4 ) or Bad genes. These data confirm that protection from development of chronic rejection is associated with expression of antiapoptotic genes in the graft.

$R T-P C R$. Using a semiquantitative competitive method of RT-PCR, we analyzed the intragraft expression of key macrophage activation products at $16 \mathrm{wk}$ after transplant, a time point when these products are maximally expressed in control animals $(12,16)$. CTLA4Ig therapy was associated with a significant decrease in intragraft expression of the $\mathrm{T}$ cell cytokines IL- 2 and TNF- $\alpha$, the macrophage cytokines IL- 6 and IL-12, the chemoattractant MCP-1, and iNOS (Fig. 5), as compared to CsA only treated controls, all previously shown to be associated with development of chronic allograft rejection in this model $(12,16)$.

These data confirm our immunohistological studies at the gene transcript level and indicate that CD28-B7 blockade late after transplantation inhibits cell-mediated and humoral immune responses associated with development of progressive chronic graft dysfunction in this model.

Intervention at 16 wk after transplant. Based on the above observations, another experimental group of animals was set up to evaluate the effect of CD28-B7 blockade at 16 wk after transplantation. In our original studies, sequential RT-PCR and immunohistological analyses of renal allografts showed that at this time period there was significant infiltration by mononuclear cells, particularly macrophages, and upregulation of key $\mathrm{T}$ cell and macrophage activation products including cytokines, chemokines, and growth factors $(12,16)$. We now show that animals treated with CTLA4Ig at 16 wk had a level of proteinuria comparable to the control group at that time point $(17.6 \pm 8.3 \mathrm{mg} / 24 \mathrm{~h}$ versus $20.8 \pm 4.1, P=\mathrm{NS})$, but, similar to the animals treated with CTLA4Ig at $8 \mathrm{wk}$, chronic graft dysfunction did not progress as evidenced by stable proteinuria at $24 \mathrm{wk} ; 24-\mathrm{h}$ protein excretion was $12.8 \pm 0.5 \mathrm{mg} / 24 \mathrm{~h}$ ( $n=3$; although numerically lower, this did not attain statistical significance versus controls because of the small number of animals in that group). However, morphologic examination showed that use of CTLA4Ig at $16 \mathrm{wk}$ reduced the extent of development of glomerulosclerosis compared to controls, such that allografts harvested at $24 \mathrm{wk}$ were comparable to $16-\mathrm{wk}$ samples, or had undergone only moderately increased focal and segmental glomerulosclerosis, whereas control grafts showed widespread, often global glomerulosclerosis (Fig. 6). Analogous reduction of development of transplant arteriosclerosis was also noted when comparing allografts in rats treated with CTLA4Ig at 16 wk versus controls (not shown).

\section{Discussion}

Clinically, chronic rejection of solid organs is the leading cause of late allograft failure. The rate of allograft loss secondary to chronic rejection has not declined in recent years, with as yet no specific therapy for this condition. Although the pathophysiology of chronic rejection remains poorly understood, several factors are known to be associated with development of this process. These factors have previously been divided into antigen-dependent and antigen-independent elements (4). Early graft injury secondary to acute rejection and/or graft ischemia appear to be the most important risk factors for development of chronic rejection. T cell recognition of alloantigen and activation are essential for initiation of the process of chronic rejection. In fact, experimental evidence suggests that graft ischemia leads to increased "immunogenicity" of the graft by 


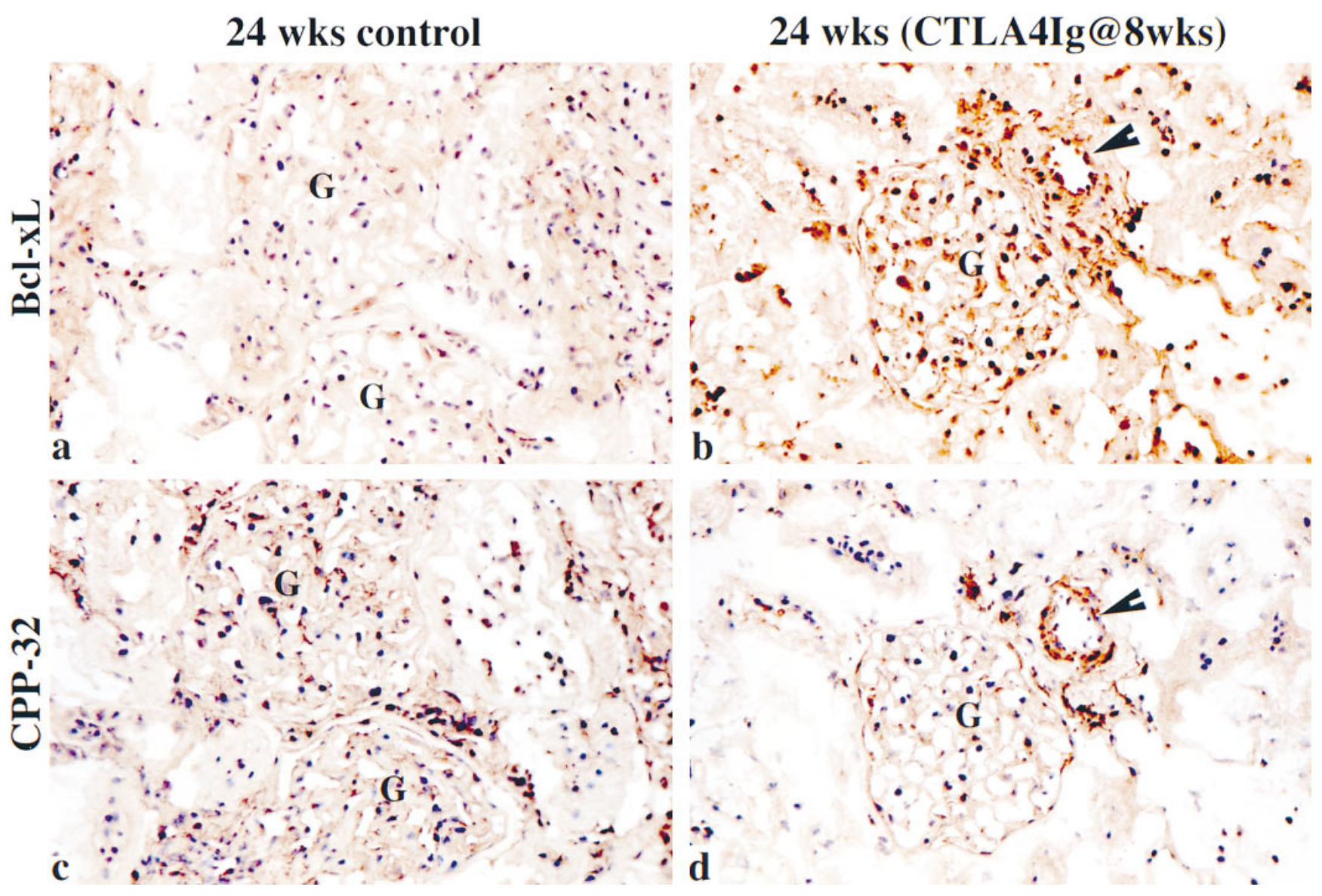

Figure 4. Contrasting expression of antiapoptotic and pro-apoptotic genes at $24 \mathrm{wk}$. Only trace expression of Bcl-xL was seen in control allografts $(a)$, whereas CTLA4Ig therapy at 8 wk led to widespread endothelial (arrow) and glomerular $(G)$ expression. Serial sections show that CPP-32 expression in each case was the reciprocal of that of Bcl-xL $(c$ and $d)$, especially with respect to glomerular staining. Note focal and segmental glomerulosclerosis in controls $(a$ and $c$ ) versus the well preserved glomerular architecture after CTLA4Ig therapy $(b$ and $d)$. Hematoxylin counterstain, $\times 400$.

upregulation of class II MHC molecule $(28,34)$ and costimulatory molecule $(28,35)$ expression. What has not been fully appreciated to date is whether chronic rejection is an irreversible consequence of initial graft injury, or whether it is an ongoing injury amenable to modification. In particular, the role of ongoing $\mathrm{T}$ cell activation in progression of chronic graft rejection remains unknown.

In this study, we have used the F344 to LEW chronic renal allograft rejection model to study the role of T cell-costimulatory activation in progression of chronic rejection. Although not fully allogeneic, this model has been extensively used to investigate mechanisms of chronic rejection (36-38). Functionally, monitoring protein excretion has been demonstrated to be the best, most sensitive surrogate marker that correlates with development of morphological changes of chronic rejection in this model; change in serum creatinine is typically a very late finding in this model (15). It has already been demonstrated that by $8-16$ wk after transplant, after acute rejection has occurred, early changes of chronic rejection can be demonstrated by subtle morphological changes and by typical expression patterns of T cell-and macrophage-activation products by immunohistology and RT-PCR $(14,16,39)$. Interestingly, in this model, chronic rejection can be reversed up to $12 \mathrm{wk}$ by retransplantation of the allograft into donor strain recipients; but not, thereafter, the process apparently progressing towards endstage organ failure (39). These findings provided the rationale for the timing of our intervention with CTLA4Ig at 8 and $16 \mathrm{wk}$ after transplantation. Our data show for the first time that $\mathrm{T}$ cell-costimulatory blockade late after transplantation results in interruption of the deterioration of graft function and prevention of development of characteristic histological features of chronic rejection. These data indicate that, once initiated, chronic rejection is not necessarily an inevitable process, but can be interrupted by the blockade of T cell-costimulatory activation, thus demonstrating a central role for $\mathrm{T}$ cell activation in the evolution of chronic graft dysfunction. We acknowledge, based on the retransplantation data (39) and the sequential morphological, immunohistological, and RT-PCR analyses data of grafts undergoing chronic rejection in this model, that blocking $\mathrm{T}$ cell-costimulatory activation pathways at a time when the chronic rejection process is far advanced and is characterized by severe arteriosclerosis, glomerulosclerosis, and extensive interstitial fibrosis will not be effective in interrupting the progression of the process. However, our data indicate that there may be a window of opportunity where specific interventions may halt the progression of chronic allograft 


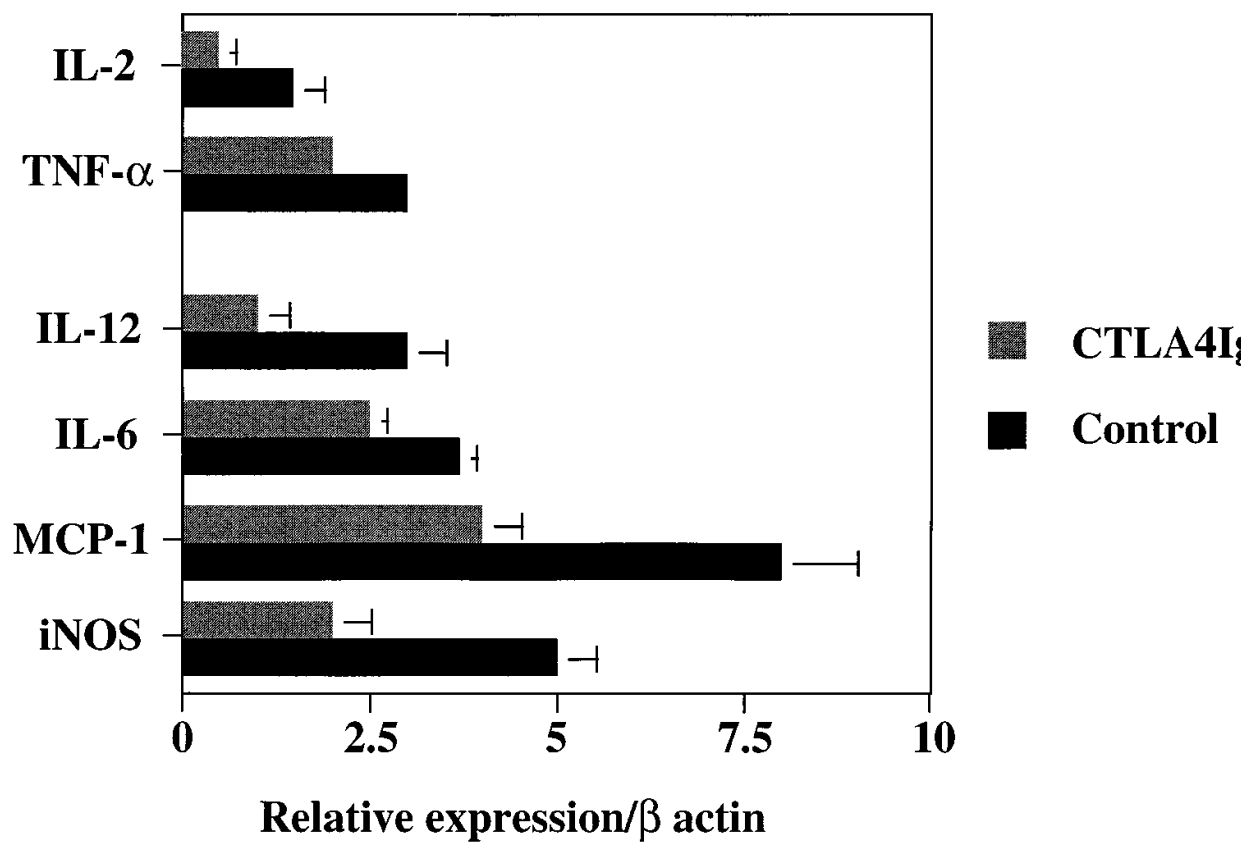

Figure 5. RT-PCR analysis of intragraft expression of $\mathrm{T}$ cell and macrophage products at $16 \mathrm{wk}$. Intragraft expression of $\mathrm{T}$ cell and macrophage products tested by RT-PCR against $\beta$-actin compared in the control group versus the group treated with CTLA4Ig at 8 wk. $P<0.05$ for all products using $t$ test for independent samples.

rejection. It is important to realize, however, that because $\mathrm{T}$ cells serve as the initiators of the effector mechanisms of the rejection process (40), it is possible that targeting such mechanisms (monocyte/macrophage/endothelial cell, or B cell activation) may also lead to interruption of chronic rejection. Our observations provide the rationale to develop novel strategies in that regard.

Clinically, transplant recipients develop progressive organ dysfunction while being maintained on immunosuppressive drugs, suggesting that alloresponses are ongoing despite maintenance immunosuppression (1). This is supported by recent observations by Batiuk et al. who showed that the calcineurin pathway is incompletely inhibited in human renal transplant recipients maintained on CsA (31). This is consistent with our recent observations in human renal transplant recipients maintained on immunosuppression, showing that the presence of peripheral blood lymphocytes primed via the indirect pathway of allorecognition may correlate with development with chronic rejection (41). More importantly, we (13) and others (10) have shown that, at least in experimental transplantation models, CsA therapy may abrogate the tolerogenic effects of $\mathrm{T}$ cellcostimulatory blockade, suggesting that CsA may interfere with regulatory mechanisms which suppress $\mathrm{T}$ cell-mediated immune responses in vivo.

The ability of CD28-B7 costimulatory blockade to interrupt an ongoing $\mathrm{T}$ cell response has been demonstrated in a model of spontaneous autoimmune lupus-like disease in mice (42), in the autoimmune encephalomyelitis model $(43,44)$, and in graft versus host disease (45). The question of whether the regulation of the $\mathrm{T}$ cell response is due to costimulatory blockade on effector or memory $\mathrm{T}$ cells remains controversial (46). In our study, the interruption of deterioration in organ dysfunction secondary to chronic rejection was brought about by a single injection of CTLA4Ig administered 8 or 16 wk after transplant. As the half-life of CTLA4Ig is only 50-70 h (47), this suggests that $\mathrm{T}$ cell-costimulatory blockade had a prolonged effect on the $\mathrm{T}$ cell response, more in keeping with an effect on memory T cells. Alternatively, CD28-B7 blockade may have resulted in generation of regulatory $\mathrm{T}$ cells that suppress ongoing cell-mediated immune responses in vivo. Little is known about whether memory $\mathrm{T}$ cells require CD28-B7-costimulatory signals in an allograft setting, although CTLA4Ig has been shown to block memory antibody production (48).

One of the known consequences of CD28-B7 signaling is the regulation of cytokine production by $\mathrm{T}$ cells $(19,20,49)$. The control group of animals show the typical upregulation of cytokines and growth factors previously noted in this model to be associated with the development of chronic rejection (12, $14,16,19,20)$. The treated group of animals had a very different profile of cytokine expression, showing downregulation of Th1 cell function and inhibition of macrophage activation. This supports previous data indicating that macrophage infiltration and subsequent tissue remodeling within the graft are dependent on $\mathrm{T}$ cell activation. It is interesting to note that IL-4 and IL-13 are upregulated, in keeping with a Th2 switch as seen in animals treated with CTLA4Ig to induce tolerance in an acute renal allograft model (50). This is contrary to the suggestion that $\mathrm{Th} 2$ cytokines, although protective against an initial acute $\mathrm{T}$ cell immune response, may lead to the development of chronic rejection because of the associated humoral immune response. In fact, in our acute rejection model, induction of tolerance to renal allografts by CTLA4Ig was associated with inhibition of donor-specific, cell-mediated (mixed lymphocyte responses and lymphocyte-mediated cytotoxicity), as well as humoral immune responses in treated animals (27, $51)$. Whether this $\mathrm{Th} 2$ switch is causally related to the protective effects of CD28-B7 blockade in this and other acute rejection models, remains undetermined (52). In fact, recent data from Lakkis et al. indicate that CTLA4Ig therapy does prolong graft survival in IL-4 knockout cardiac transplant recipients (53), although the extent to which long-term survival was associated with development of chronic rejection was not examined in that model.

Given that the brunt of chronic rejection involves progres- 

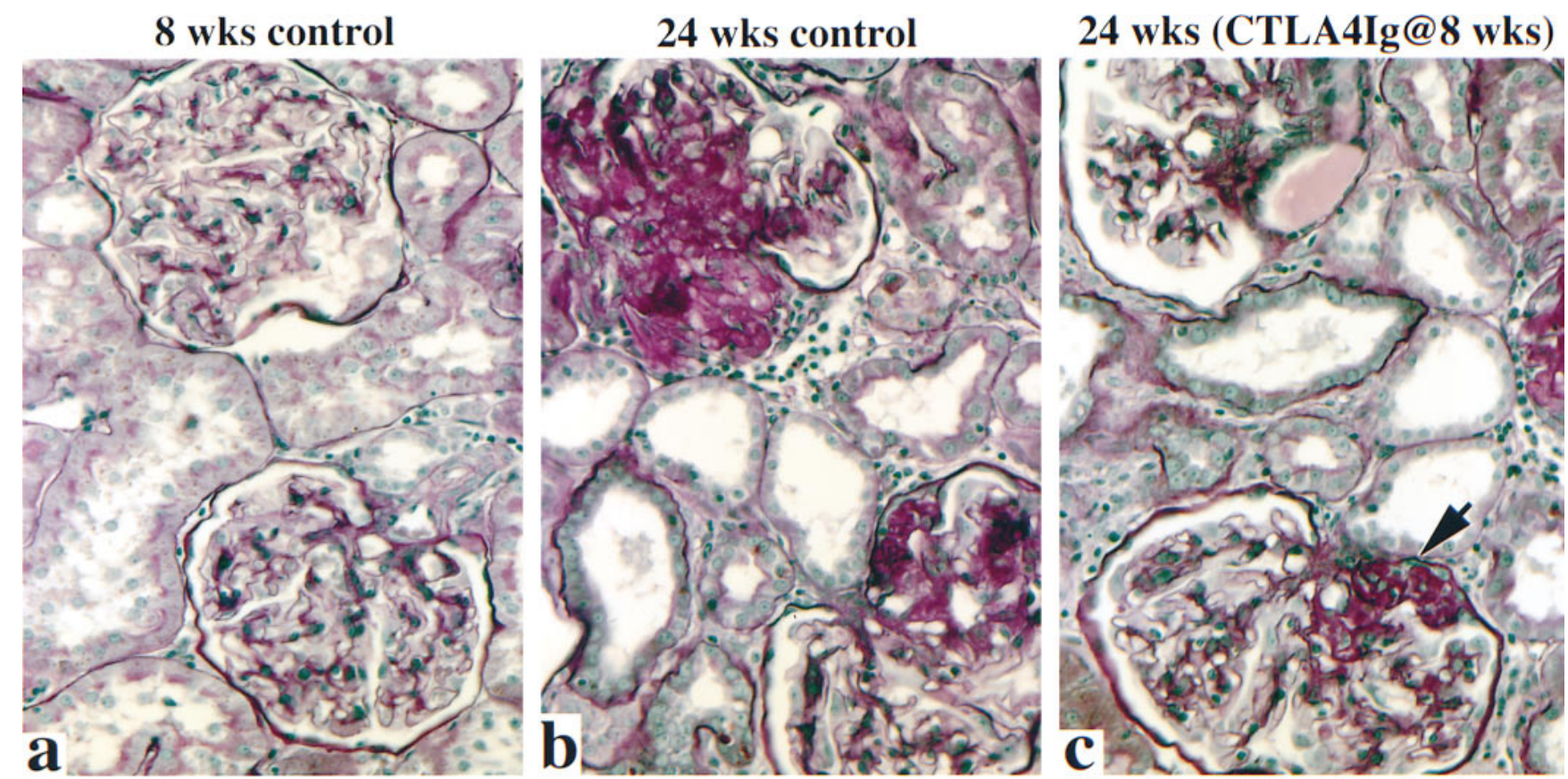

\section{6 wks control}

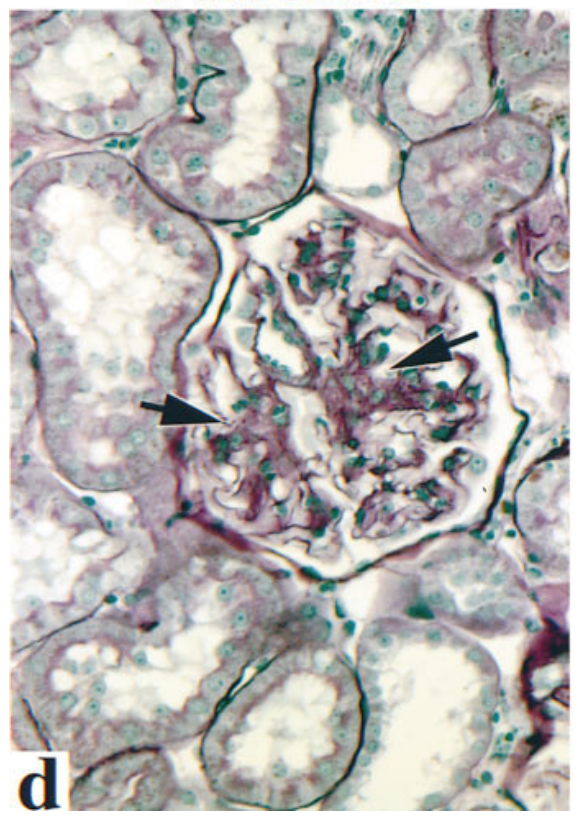

\section{4 wks control}

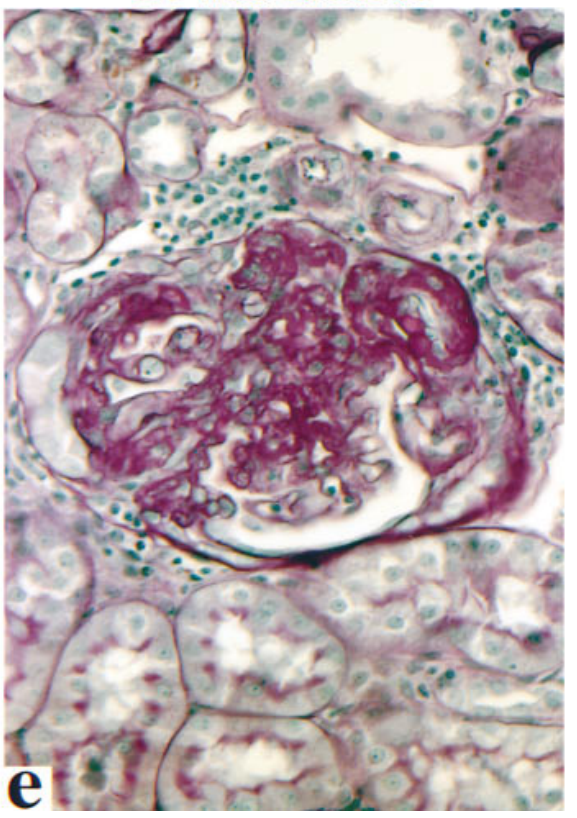

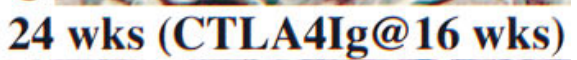

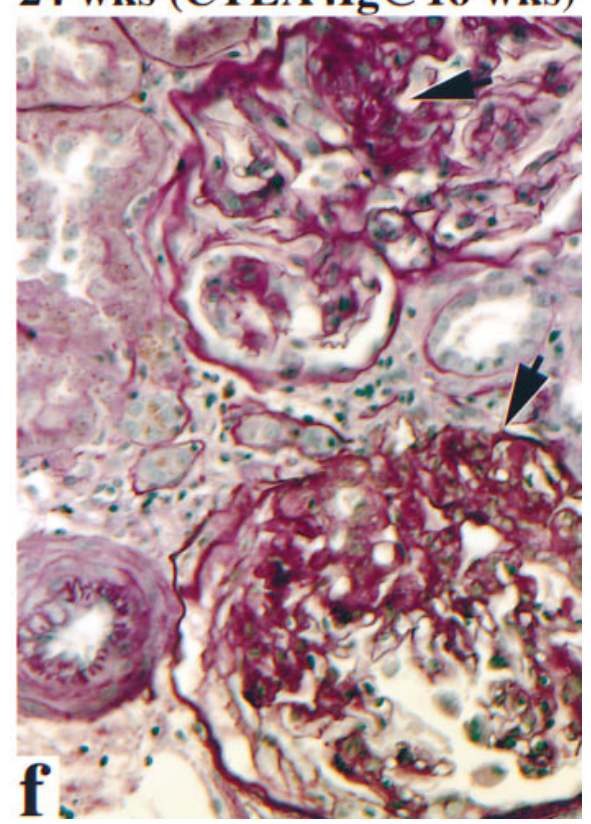

Figure 6. PAS staining of renal allografts showing the extent of global or focal (arrows) glomerulosclerosis and its reduction depending upon the timing of use of CTLA4Ig. Therapy with CTLA4Ig at $8 \mathrm{wk}(a)$ markedly inhibited development of glomerulosclerosis, with only mild proliferative lesions apparent at $24 \mathrm{wk}(c)$, in contrast to controls $(b)$. In animals in which therapy was delayed to 16 wk $(d-f)$, most glomeruli already showed mild to moderate focal and segmental glomerulosclerosis $(d)$, but whereas controls developed severe glomerulosclerosis by 24 wk $(e)$, CTLA4Ig-treated recipients still showed only mild to moderate proliferative lesions (arrows) with numerous patent capillary loops apparent within each glomerulus comparable to the lesions seen at $16 \mathrm{wk}$. PAS staining, $\times 200$.

sive injury to graft vessels and subsequent proliferative and fibrotic responses, the expression of protective genes (30) in CTLA4Ig-treated recipients was of interest. Protective genes, such as Bcl-2 and Bcl-xL, regulate CPP-32-induced apoptosis, and inhibit the induction of multiple pro-inflammatory genes whose expression is associated with activation of the transcription factor, NF-кB (33). The extent to which protective gene expression is attributable to the actions of Th2 cytokines alone or in combination with other immunoregulatory molecules is under investigation, but the current studies extend evidence of their expression to orthotopic, functional transplant models, which will facilitate the further dissection of their mechanisms of protection in vitro and in vivo $(30,31)$.

In summary, we show that progression of chronic graft dysfunction is $\mathrm{T}$ cell dependent, and that strategies targeted at $\mathrm{T}$ cell-costimulatory blockade may provide a novel approach to 
prevent and interrupt this process. Obviously, such strategies have to be proven effective in fully allogeneic small and large animal models, particularly primates (32), before clinical development for human organ transplant recipients.

\section{Acknowledgments}

This study was supported by National Institutes of Health (Bethesda, MD) grants PO1-AI40152-01, R01-AI40629-01, and RO1-AI4229901. Mohamed H. Sayegh is recipient of the National Kidney Foundation Clinical Scientist Award. Anil Chandraker is a recipient of the Juvenile Diabetes Foundation International Fellowship Award.

\section{References}

1. Carpenter, C.B. 1995. Long-term failure of renal transplants: adding insult to injury. Kidney Int. 50:S40-S44.

2. Hostetter, T.H. 1994. Chronic transplant rejection. Kidney Int. 46:266-279.

3. Paul, L.C. 1995. Experimental models of chronic renal allograft rejection. Transplant. Proc. 27:2126-2128.

4. Tullius, S.G., and N.L. Tilney. 1995. Both alloantigen-dependent and -independent factors influence chronic allograft rejection. Transplantation (Baltimore). 59:313-318.

5. Shin, Y.T., D.H. Adams, L.R. Wyner, E. Akalin, M.H. Sayegh, and M.J. Karnovsky. 1995. Intrathymic tolerance in the Lewis-to-F344 chronic cardiac allograft rejection model. Transplantation (Baltimore). 59:1647-1653.

6. Orloff, M.S., E.M. DeMara, M.L. Coppage, N. Leong, M.A. Fallon, J. Sickel, X. Zuo, J. Prehn, and S.C. Jordan. 1995. Prevention of chronic rejection and graft arteriosclerosis by tolerance induction. Transplantation (Baltimore). 59:282-288.

7. Colson, Y.L., K. Zadach, M. Nalesnik, and S.T. Ildstad. 1995. Mixed allogeneic chimerism in the rat: donor-specific transplantation tolererance without chronic rejection for primarily vascularized cardiac allografts. Transplantation (Baltimore). 60:971-980.

8. Murase, N.M., T.E. Starzl, M. Tanable, S. Fujisaki, H. Miyazawa, Q. Ye, C.P. Delaney, J.J. Fung, and A.J. Demetris. 1995. Variable chimerism, graft versus-host disease, and tolerance after different kinds of cell and whole organ transplantation from Lewis to Brown Norway rats. Transplantation (Baltimore). 60:158-171.

9. Russell, M.E., W.W. Hancock, E. Akalin, A.F. Wallace, T. GlysingJensen, T. Willett, and M.H. Sayegh. 1996. Chronic cardiac rejection in the Lewis to F344 rat model: blockade of CD28-B7 costimulation by CTLA4Ig modulates $\mathrm{T}$ cell and macrophage activation and attenuates arteriosclerosis. $J$. Clin. Invest. 97:833-838.

10. Larsen, C.P., E.T. Alwood, D.Z. Alexander, S.C. Ritchie, R. Hendrix, C. Tucker-Byrden, H.R. Cho, A. Aruffo, D. Hollenbaugh, P.S. Linsley, K.J. Winn, and T.C. Pearson. 1996. Long-term acceptance of skin and cardiac allografts after blocking CD40 and CD28 pathways. Nature. 381:434-438.

11. Sayegh, M.H., X.-Z. Zheng, C. Magee, W.W. Hancock, and L.A. Turka. 1997. Donor antigen is necessary for the prevention of chronic rejection in CTLA4Ig-treated murine cardiac allografts. Transplantation (Baltimore). 64: $1646-1650$.

12. Azuma, H., A. Chandraker, K. Nadeau, W.W. Hancock, C.B. Carpenter, N.L. Tilney, and M.H. Sayegh. 1996. Blockade of T-cell costimulation prevents development of experimental chronic renal allograft rejection. Proc. Natl. Acad. Sci. USA. 93:12439-12444.

13. Chandraker, A., M.E. Russell, T. Glysing-Jensen, T.A. Willett, and M.H. Sayegh. 1997. T cell costimulatory blockade in experimental chronic cardiac allograft rejection: effects of cyclosporine and donor antigen. Transplantation (Baltimore). 63:1053-1058.

14. Hancock, W.W., W.M. Whitley, S.G. Tullius, U.W. Heeman, B. Wasowska, W.M. Baldwin III, and N.L. Tilney. 1993. Cytokines, adhesion molecules, and the pathogenesis of chronic rejection of rat renal allografts. Transplantation (Baltimore). 56:643-650.

15. Diamond, J.R., N.L. Tilney, J. Frye, G. Ding, J. McElroy, I. Pesek-Diamon, and H. Yang. 1993. Progressive albuminuria and glomerulosclerosis in a rat model of chronic renal allograft rejection. Transplantation (Baltimore). 54: $710-716$

16. Nadeau, K.C., H. Azuma, and N.L. Tilney. 1995. Sequential cytokine dynamics in chronic rejection of rat renal allografts: key roles for RANTES and MCP-1. Proc. Natl. Acad. Sci. USA. 92:8729-8733.

17. Linsley, P.S., and J.A. Ledbetter. 1993. The role of the CD28 receptor during T cell responses to antigen. Ann. Rev. Immunol. 11:191-212.

18. June, C.H., J.A. Bluestone, L.M. Nadler, and C.B. Thompson. 1994. The B7 and CD28 receptor families. Immunol. Today. 15:321-331.

19. Bluestone, J.A. 1995. New perspectives of CD28-B7-mediated T cell costimulation. Immunity. 2:555-559.
20. Thompson, C.B. 1995. Distinct roles for the costimulatory ligands B7-1 and B7-2 in T helper cell differentiation. Cell. 81:979-982.

21. Lenschow, D.J., Y. Zeng, J.R. Thistlethwaite, A. Montag, W. Brady, M.G. Gibson, P.S. Linsley, and J.A. Bluestone. 1992. Long-term survival of xenogeneic pancreatic islet grafts induced by CTLA4lg. Science. 257:789-792.

22. Turka, L.A., P.S. Linsley, H. Lin, W. Brady, J.M. Leiden, R.Q. Wei, M.L. Gibson, X.G. Zheng, S. Myrdal, D. Gordon, T. Bailey, S.F. Bolling, and C.B. Thompson. 1992. T-cell activation by the CD28 ligand B7 is required for cardiac allograft rejection in vivo. Proc. Natl. Acad. Sci. USA. 89:11102-11105.

23. Lin, H., S.F. Bolling, P.S. Linsley, R.Q. Wei, D. Gordon, C.B. Thompson, and L.A. Turka. 1993. Long-term acceptance of major histocompatibility complex mismatched cardiac allografts induced by CTLA4 Ig plus donor-specific transfusion. J. Exp. Med. 178:1801-1806.

24. Pearson, T.C., D.Z. Alexander, K.J. Winn, P.S. Linsley, R.P. Lowry, and C.P. Larsen. 1994. Transplantation tolerance induced by CTLA4-Ig. Transplantation (Baltimore). 57:1701-1706.

25. Pearson, T., D. Alexander, R. Hendrix, E. Elwood, P.S. Linsley, K. Winn, and C. Larsen. 1995. CTLA4-Ig plus bone marrow induces long-term allograft survival and donor specific unresponsiveness in the murine model. Evidence for hematopoietic chimerism. Transplantation (Baltimore). 61:997-1004.

26. Perico, N., O. Imberti, M. Bontempelli, and G. Remuzzi. 1995. Toward novel antirejection strategies: in vivo immunosuppressive properties of CTLA4Ig. Kidney Int. 47:241-246.

27. Sayegh, M.H., E. Akalin, W.W. Hancock, M.E. Russell, C.B. Carpenter, and L.A. Turka. 1995. CD28-B7 blockade after alloantigenic challange in vivo inhibits Th1 cytokines but spares Th2. J. Exp. Med. 181:1869-1874.

28. Takada, M., K.C. Nadeau, G.D. Shaw, K.A. Marquette, and N.L. Tilney. 1997. The cytokine-adhesion molecule cascade in ischemia/reperfusion injury of the rat kidney inhibition by a soluble P-selectin ligand. J. Clin. Invest. 99: 2682-2690.

29. Takada, M., A. Chandraker, K.C. Nadeau, M.H. Sayegh, and N.L. Tilney. 1997. The role of B7 costimulatory pathway in experimental cold ischemia/ reperfusion injury. J. Clin. Invest. 100:1199-1203.

30. Bach, F.H., C. Ferran, P. Hechenleitner, W. Mark, N. Koyamada, T. Miyatake, H. Winkler, A. Badrichani, D. Candinas, and W.W. Hancock. 1997. Accommodation of vascularized xenografts: expression of "protective genes" by donor endothelial cells in a host Th2 cytokine environment. Nat. Med. 3:196204

31. Batiuk, T.D., F. Pazderka, J. Enns, C.L. De, and P.F. Halloran. 1996 Cyclosporine inhibition of leukocyte calcineurin is much less in whole blood than in culture medium. Transplantation (Baltimore). 61:158-161.

32. Hancock, W.W. 1998. Impact of alloimmunity on chronic tissue remodelling: role of cytokines and protective genes in the vessel wall. Transplant. Immunol. 5:277-281.

33. Vella, J.P., M. Ferreira, B. Murphy, S.I. Alexander, W. Harmon, C.B Carpenter, and M.H. Sayegh. 1997. Indirect allorecognition of MHC allopeptides in human renal transplant recipients with chronic graft dysfunction. Transplantation (Baltimore). 64:795-800.

34. Goes, N., J. Urmson, V. Ramassar, and P.F. Halloran. 1995. Ischemic acute tubular necrosis induces an extensive local cytokine response: evidence for induction of interferon-g, transforming growth factor b-1, granulocyte-macrophage colony stimulating factor, interleukin-2 and interleukin-10. Transplantation (Baltimore). 59:565-572.

35. Chandraker, A., M. Takada, K.C. Nadeau, R. Peach, N.L. Tilney, and M.H. Sayegh. 1997. CD28-B7 blockade in organ dysfunction secondary to cold ischemia/reperfusion. Kidney Int. 52:1678-1684.

36. Shihab, F.S., A.M. Tanner, Y. Shao, and M.I. Weffer. 1996. Expression of TGF-beta 1 and matrix proteins is elevated in rats with chronic rejection. Kidney Int. 50:1904-1913.

37. Benediktsson, H., R. Chea, A. Davidoff, and L.C. Paul. 1996. Antihypertensive drug treatment in chronic renal allograft rejection in the rat. Effect on structure and function. Transplantation (Baltimore). 62:1634-1642.

38. Tullius, S.G., M. Nieminen, W.O. Bechstein, S. Jonas, T. Steinmuller, J. Pratschke, K. Zeilinger, E. Graser, H.D. Volk, and P. Neuhaus. 1997. Chronically rejected rat kidney allografts induce donor-specific tolerance. Transplantation (Baltimore). 64:158-161.

39. Tullius, S.G., W.W. Hancock, U. Heemann, H. Azuma, and N.L. Tilney. 1994. Reversibility of chronic renal allograft rejection. Critical effect of time after transplantation suggests both host immune dependent and independent phases of progressive injury. Transplantation (Baltimore). 58:93-99.

40. Sayegh, M.H., and L.A. Turka. 1998. The role of T cell costimulatory activation in transplant rejection. N. Engl. J. Med. In press.

41. Kirk, A., D. Harlan, N. Armstrong, T. Davis, Y. Dong, G. Gray, X Hong, D. Thomas, J. Fechner, and S. Knechtle. 1997. CTLA4-Ig and anti-CD40 ligand prevent renal allograft rejection in primates. Proc. Natl. Acad. Sci. USA. 94:8789-8794

42. Finck, B.K., P.S. Linsley, and D. Wofsy. 1994. Treatment of murine lupus with CTLA4Ig. Science. 265:1225-1227.

43. Miller, S.D., C.L. Vanderlugt, D.J. Lenschow, J.G. Pope, N.J. Karandikar, M.C. Dal Canto, and J.A. Bluestone. 1995. Blockade of CD28/B7-1 interaction prevents epitope spreading and clinical relapses of murine EAE. Immunity. 3:739-745. 
44. Khoury, S.J., A. Akalin, A. Chandraker, L.A. Turka, P.S. Linsley, M.H. Sayegh, and W.W. Hancock. 1995. CD28-B7 costimulatory blockade by CTLA4Ig prevents actively induced experimental autoimmune encephalomyelitis and inhibits Th1 but spares Th2 cytokines in the central nervous system. $J$. Immunol. 155:4521-4524.

45. Via, C.S., V. Rus, P. Nguyen, P. Linsley, and W.C. Gause. 1996. Differential effect of CTLA4Ig on murine graft-versus-host disease (GVHD) development: CTLA4Ig prevents both acute and chronic GVHD development but reverses only chronic GVHD. J. Immunol. 157:4258-4267.

46. Gause, W.C., V. Mitro, C. Via, P. Linsley, J.J. Urban, and R.J. Greenwald. 1997. Do effector and memory T helper cells also need B7 ligand costimulatory signals? J. Immunol. 159:1055-1058.

47. Srinivas, N.R., R.S. Weiner, W.C. Shyu, J.D. Calore, D. Tritschler, L.K. Tay, J.S. Lee, D.S. Greene, and R.H. Barbhaiya. 1996. A pharmacokinetic study of intravenous CTLA4Ig, a novel immunosuppressive agent, in mice. $J$. Pharm. Sci. 85:296-298.

48. Linsley, P.S., P.M. Wallace, J. Johnson, M.G. Gibson, J.L. Greene, J.A. Ledbetter, C. Singh, and M.A. Tepper. 1992. Immunosuppression in vivo by a soluble form of the CTLA-4 T cell activation molecule. Science. 257:792-795.
49. Herold, K.C., J. Lu, I. Rulifson, V. Vezys, D. Taub, M.J. Grubsby, and J.A. Bluestone. 1997. Regulation of C-C chemokine production by murine $\mathrm{T}$ cells by CD28-B7 costimulation. J. Immunol. 159:4150-4153.

50. Sayegh, M.H., E. Akalin, W.W. Hancock, and M.E. Russell. 1994. Blocking the CD28-B7 costimulatory T cell activation pathways with CTLA-4 $\mathrm{Ig}$ presents chronic rejection in the Lew to F344 vascularized rat cardiac allograft model. J. Am. Soc. Nephrol. 5:989.

51. Akalin, E., A. Chandraker, M.E. Russell, L.A. Turka, W.W. Hancock, and M.H. Sayegh. 1996. CD28-B7 T cell costimulatory blockade by CTLA4Ig in the rat renal allograft model: inhibition of cell-mediated and humoral immune responses in vivo. Transplantation (Baltimore). 62:1942-1945.

52. Nickerson, P., J. Steiger, X.X. Zheng, A.W. Steele, W. Steurer, C.P. Roy, and T.B. Strom. 1997. Manipulation of cytokine networks in transplantation: false hope or realistic opportunity for tolerance? Transplantation (Baltimore). 63:489-494.

53. Lakkis, F., B. Konieczny, S. Saleem, F. Baddoura, P. Linsley, D. Alexander, R. Lowry, T. Pearson, and C. Larsen. 1997. Blocking the CD28-B7 T cell costimulation pathway induces long term cardiac allograft acceptance in the absence of IL-4. J. Immunol. 158:2443-2448. 